\title{
Quandle Coloring Quivers of Surface-Links
}

\author{
Jieon Kim* $\quad$ Sam Nelson ${ }^{\dagger} \quad$ Minju Seo ${ }^{\ddagger}$
}

\begin{abstract}
Quandle coloring quivers are directed graph-valued invariants of oriented knots and links, defined using a choice of finite quandle $X$ and set $S \subset \operatorname{Hom}(X, X)$ of endomorphisms. From a quandle coloring quiver, a polynomial knot invariant known as the in-degree quiver polynomial is defined. We consider quandle coloring quiver invariants for oriented surface-links, represented by marked graph diagrams. We provide example computations for all oriented surface-links with ch-index up to 10 for choices of quandles and endomorphisms.
\end{abstract}

KEYWORDS: Quandle coloring quivers, surface-links, in-degree polynomial.

2020 MSC: $57 \mathrm{~K} 12$

\section{Introduction}

In [5], a diagrammatic approach to the study of knotted and linked surfaces in $\mathbb{R}^{4}$ using knotted 4-valent graphs with decorated vertices was introduced. Every compact surface embedded in $\mathbb{R}^{4}$ can be positioned by ambient isotopy to have all its maxima with respect to an axis $w$ at $w=1$, all of its minima at $w=-1$ and all of its saddle points at $w=0$; this is known as a hyperbolic splitting. Then the cross-section of the surface with the $w=0$ hyperplane is a knotted 4-valent graph with the properties that (1) each vertex represents a saddle point and (2) smoothing the vertices by taking a slightly higher or lower cross-section in the $w$ direction results in an unlink. These diagrams, known as marked graph diagrams or marked vertex diagrams or ch-diagrams, determine surface-links up to ambient isotopy. The combinatorial moves on marked graph diagrams encoding ambient isotopy in $\mathbb{R}^{4}$ are known as Yoshikawa moves; in [4, the first listed author and coauthors discuss the history of these moves and provide generating sets of the moves.

Quandles are algebraic structures with axioms defined from the Reidemeister moves of classical knot knot theory. Given a finite quandle $X$, the set of quandle homomorphisms from the fundamental quandle of a classical knot to $X$ is an invariant, and its cardinality is known as the quandle counting invariant. See [2] and the references therein for more.

In [1], the second-listed author and a coauthor defined an enhancement of the quandle counting invariant for classical knots, the quandle coloring quiver. This quiver-valued invariant of classical knots and links categorifies the quandle counting invariant, and the extra information in the quiver can distinguish knots with the same counting invariant. Since dealing with large quivers directly can be problematic, further invariants were defined, notably the in-degree polynomial.

Quandle-based invariants have been defined for surface-links and studied in papers such as [3, 4]. In this paper we define the quandle coloring quiver for surface-links. The paper is organized as follows. In Section 2 we review the basics of quandles and of surface-links. In Section 3 we define quandle coloring quivers for surface-links and provide examples and computations. In particular we show that the new invariant is a proper enhancement in the sense that it can distinguish surface-links with the same quandle counting invariant. We further extend the in-degree polynomial to the case of surface-links. We conclude in Section 4 with some questions for future work.

*mail: jieonkim7@gmail.com. Supported by Young Researchers Program through the National Research Foundation of Korea (NRF), funded by the Ministry of Education, Science and Technology (NRF-2018R1C1B6007021).

${ }^{\dagger}$ Email: Sam.Nelson@cmc.edu. Partially supported by Simons Foundation collaboration grant 316709

$\ddagger$ Email: mingzuu@pusan.ac.kr 


\section{Quandles and Surface-Links}

In this section, we review surface-links, marked graph diagrams and quandles. We begin with the basics of quandle theory. See [2] and the references therein for more detail.

Definition 1. A set $X$ equipped with a binary operation $\triangleright$ is a quandle if it satisfies

1. $x \triangleright x=x$ for all $x \in X$,

2. for each $y \in X$, the map $f_{y}: X \rightarrow X$ defined by $f_{y}(x)=x \triangleright y$ is a bijection, and

3. $(x \triangleright y) \triangleright z=(x \triangleright z) \triangleright(y \triangleright z)$ for all $x, y, z \in X$.

Example 1. Let $K$ be an oriented classical knot. The fundamental quandle or knot quandle of $K$, denoted $Q(K)$, is the quandle generated by generators corresponding to arcs in a diagram of $K$ with the relation

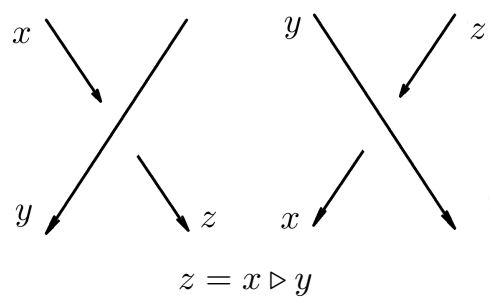

at each crossing. More formally, elements of the knot quandle are equivalence classes of quandle words in these generators modulo the equivalence relation generated by the quandle axioms and the crossing relations. There is also a geometric interpretation of the knot quandle in terms of path homotopy classes of paths from a basepoint to the boundary torus of the knot complement; see [2] and the references therein for more detail.

Example 2. Let $G$ be a group. $G$ has several quandle structures including:

- Core quandles. Setting

$$
x \triangleright y=x y^{-1} x
$$

defines a quandle structure on any group. In the case of a cyclic group $G=\mathbb{Z}_{n}$ written additively, this structure is sometimes called a cyclic quandle and has the operation

$$
x \triangleright y=2 y-x .
$$

The famous Fox colorings of knots are really homomorphisms from the knot quandle to the core quandle of $\mathbb{Z}_{3}$.

- Conjugation quandles. For any integer $n \in \mathbb{Z}$, setting

$$
x \triangleright y=y^{-n} x y^{n}
$$

defines a quandle structure on $G$. The case $n=1$ for $G$ the knot group of a knot is closely related to the knot quandle, and the cases $n \geq 2$ are closely related to the Wada groups of a knot, the $n=2$ case of which was shown to be a complete invariant for classical knots in [6]. The $n=0$ case (or indeed, any $n$ for $G$ abelian) is called the trivial quandle on $n$ elements.

Example 3. Let $X$ be any module over $\mathbb{Z}\left[t^{ \pm 1}\right]$. Then $X$ is a quandle with

$$
x \triangleright y=t x+(1-t) y
$$


known as an Alexander quandle. The Fundamental Alexander quandle of a classical knot, with a generator for each arc and the relation

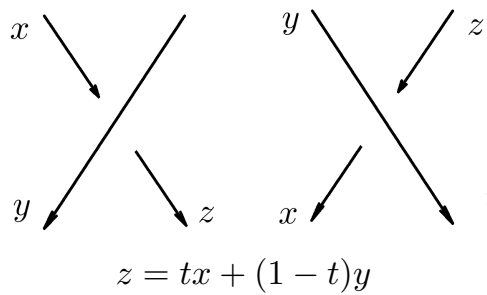

for each crossing, is the Alexander module of the knot considered as a quandle. As such, it determines the Alexander polynomials of the knot.

Example 4. Let $V$ be a vector space over a field $\mathbb{F}$ and [,] $: V \times V \rightarrow \mathbb{F}$ a symplectic form. Then $V$ is a quandle, known as a symplectic quandle, under the operation

$$
\vec{x} \triangleright \vec{y}=\vec{x}+[\vec{x}, \vec{y}] \vec{y} .
$$

Example 5. Any quandle structure on a finite set $X=\{1,2, \ldots, n\}$ can be expressed via an operation table. For example, $\operatorname{Core}\left(\mathbb{Z}_{3}\right)$ has the operation table

\begin{tabular}{l|lll}
$\triangleright$ & 1 & 2 & 3 \\
\hline 1 & 1 & 3 & 2 \\
2 & 3 & 2 & 1 \\
3 & 2 & 1 & 3.
\end{tabular}

Definition 2. Let $X, Y$ be quandles with multiplication operations indicated by $\triangleright_{X}$ and $\triangleright_{Y}$ respectively. A map $f: X \rightarrow Y$ is a quandle homomorphism given that $f\left(a \triangleright_{X} b\right)=f(a) \triangleright_{Y} f(b)$ for any $a, b \in X$.

Definition 3. Let $L$ be an oriented knot or link and $X$ a finite quandle called the coloring quandle. The coloring space $\operatorname{Hom}(\mathcal{Q}(L), X)$ is the space of quandle homomorphisms from $\mathcal{Q}(L) \rightarrow X$. The quandle counting invariant is the cardinality of the coloring space, $|\operatorname{Hom}(\mathcal{Q}(L), X)|$, which we will denote by $\Phi_{X}^{\mathbb{Z}}(L)$.

We now recall the basics of surface-links.

Definition 4. A surface-link is a closed surface smoothly embedded in $\mathbb{R}^{4}$. If a surface-link is (orientable and) oriented, then we call it an oriented surface-link.

Two surface-links $F$ and $F^{\prime}$ are said to be equivalent if there exists an orientation-preserving homeomorphism $h: \mathbb{R}^{4} \rightarrow \mathbb{R}^{4}$ such that $h(F)=F^{\prime}$. When $F$ and $F^{\prime}$ are oriented, it is assumed that $\left.h\right|_{F}: F \rightarrow F^{\prime}$ is an orientation preserving homeomorphism. An equivalence class of a surface-link is called a surface-link type. A surface-knot is trivial(or unknotted) if it is obtained from some standard surfaces in $\mathbb{R}^{4}$ (i.e. a standard 2 sphere, a standard torus, and standard projective planes in $\mathbb{R}^{4}$ ) by taking a connected sum. A surface-link is trivial(or unknotted) if it is obtained from some trivial surface-knots by taking a split union.

Definition 5. A marked vertex graph or simply a marked graph is a spatial graph $G$ in $\mathbb{R}^{3}$ which satisfies that $G$ is a finite regular graph possibly with 4 -valent vertices, say $v_{1}, v_{2}, \ldots, v_{n}$; each vertex $v_{i}$ is a rigid vertex (that is, we fix a rectangular neighborhood $N_{i}$ homeomorphic to $\{(x, y) \mid-1 \leq x, y \leq 1\}$, where $v_{i}$ corresponds to the origin and the edges incident to $v_{i}$ are represented by $x^{2}=y^{2}$ ); each vertex $v_{i}$ has a marker which is the interval on $N_{i}$ given by $\left\{(x, 0) \mid-\frac{1}{2} \leq x \leq \frac{1}{2}\right\}$.

Definition 6. An orientation of a marked graph $G$ is a choice of an orientation for each edge of $G$ in such a way that every vertex in $\mathrm{G}$ looks like or A marked graph is said to be orientable if it admits an orientation. By an oriented marked graph, we mean an orientable marked graph with a fixed orientation. 
Definition 7. If two oriented marked graphs are ambient isotopic in $\mathbb{R}^{3}$ with keeping rectangular neighborhoods, an orientation and markers, they are equivalent.

Definition 8. For any surface-link $F$, there exists a surface-link $F^{\prime}$ satisfying the following:

1. $F^{\prime}$ is equivalent to $F$ and has only finitely many Morse's critical points,

2. all maximal points of $F^{\prime}$ lie in $\mathbb{R}_{1}^{3}$,

3. all minimal points of $F^{\prime}$ lie in $\mathbb{R}_{-1}^{3}$,

4. all saddle points of $F^{\prime}$ lie in $\mathbb{R}_{0}^{3}$.

We call a representation $F^{\prime}$ in the previous theorem, a hyperbolic splitting of $F$.

Definition 9. The zero section $\mathbb{R}_{0}^{3} \cap F^{\prime}$ of the hyperbolic splitting $F^{\prime}$ gives us a 4 -valent graph. We assign to each vertex a marker that informs us about one of the two possible types of saddle points. Then we obtain a diagram representing $F$, which is called a marked graph diagram of $F$.

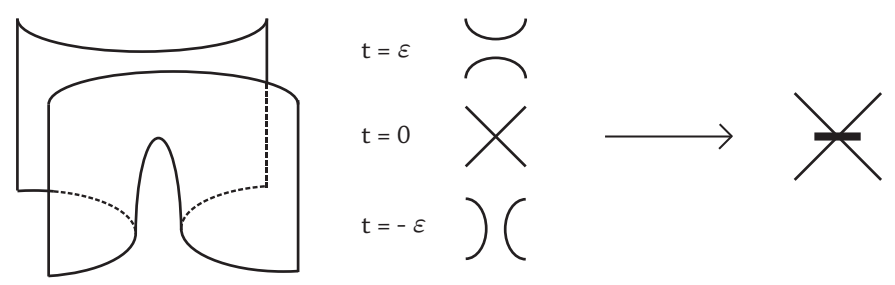

Definition 10. An oriented marked graph $G$ in $\mathbb{R}^{3}$ can be described as usual by a diagram $D$ in $\mathbb{R}^{2}$, which is an oriented link diagram in $\mathbb{R}^{2}$ possibly with some marked 4 -valent vertices whose incident four edges have orientations illustrated as above, and is called an oriented marked graph diagram of $G$.

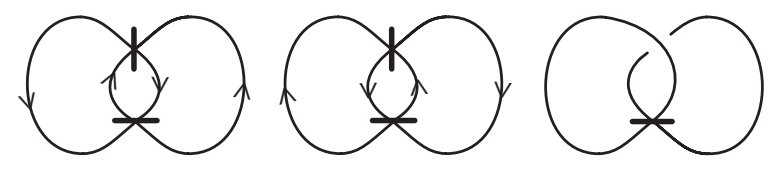

Definition 11. A (oriented) marked graph diagram $\mathrm{D}$ is admissible if both resolutions $L_{+}(D)$ and $L_{-}(D)$ are link diagrams of trivial links.
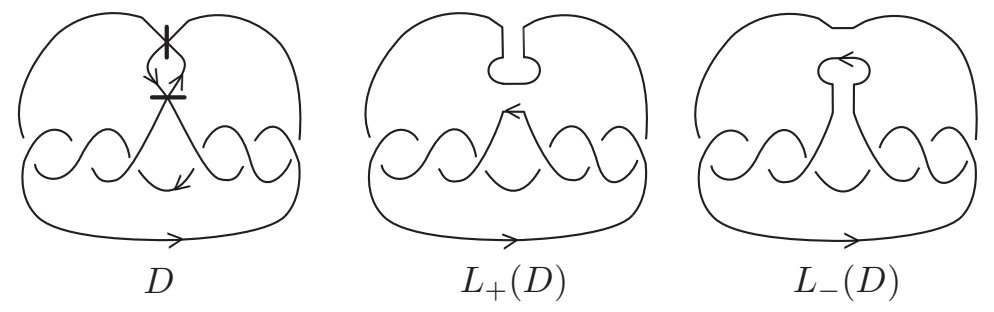

Given an admissible diagram, we can connect the two smoothings with a surface with saddle points where marked vertices were and cap off the unlinked components to obtain a broken surface diagram of the surface-link. 
Example 6. The pictured marked graph diagram determines the pictured surface-link.
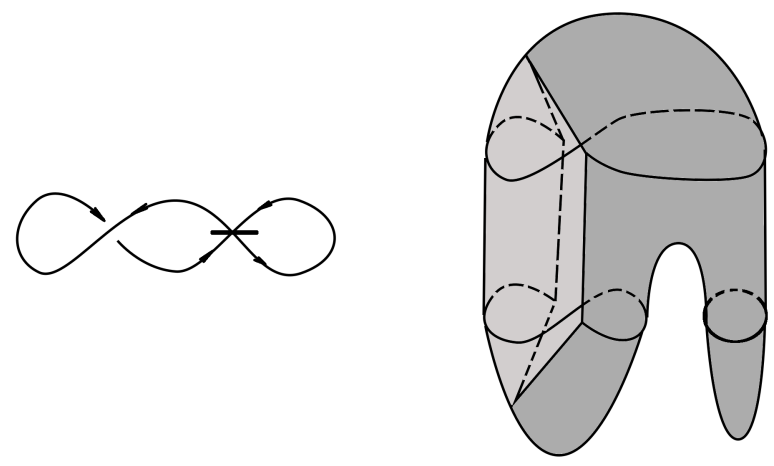

In summary, we have the following:

Theorem 1. (See [3, 4, 5] for more details)

1. For an admissible marked graph diagram $D$, there is a surface-link $L$ represented by $D$.

2. Let $L$ be a surface-link. Then there is an admissible marked graph diagram $D$ such that $L$ is represented by $D$.

Remark 1. We note that non-admissible marked vertex diagrams represent not closed surface-links but cobordisms between the classical links given by the upper and lower smoothings.

The combinatorial moves on marked graph diagrams capturing ambient isotopy of surface-links are known as the Yoshikawa moves. In addition to the three classical Reidemeister moves, one generating set of oriented Yoshikawa moves is:
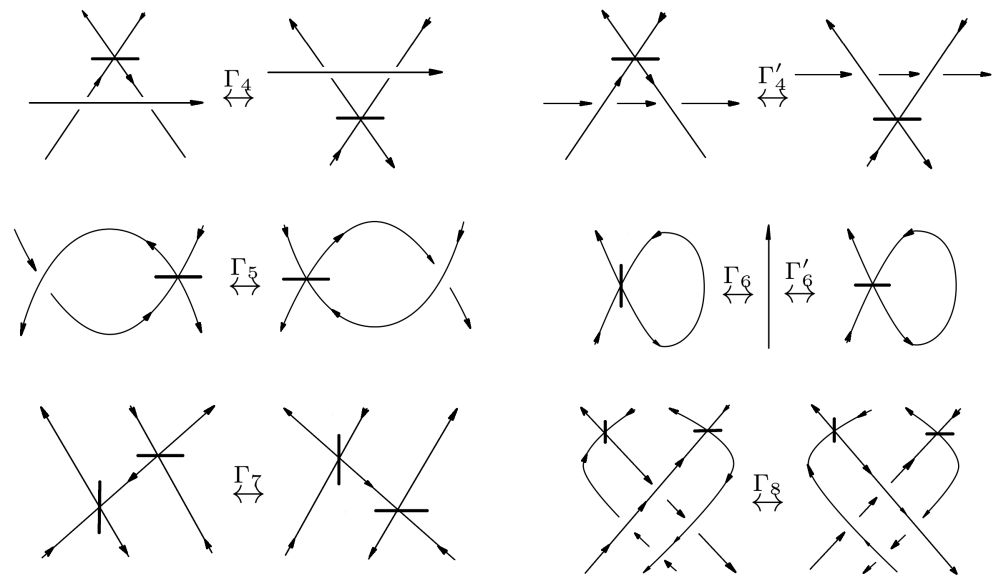

See 4] for more.

\section{Quandle Coloring Quivers of Surface-Links}

Next we recall some facts about quandle coloring quivers. See [1] for more. 
Definition 12. Let $X$ be a finite quandle and $L$ an oriented link. For any set of quandle endomorphisms $S \subset \operatorname{Hom}(X, X)$, the associated quandle coloring quiver, denoted $Q_{X}^{S}(L)$, is the directed graph with a vertex for every element $f \in \operatorname{Hom}(\mathcal{Q}(L), X)$ and an edge directed from $f$ to $g$ when $g=\phi f$ for an element $\phi \in S$. Important special cases include the case $S=\operatorname{Hom}(X, X)$, which we call the full quandle coloring quiver of $L$ with respect to X, denoted $Q_{X}(L)$, and the case when $S=\{\phi\}$ is a singleton, which we will denote by $Q_{X}^{\phi}(L)$.

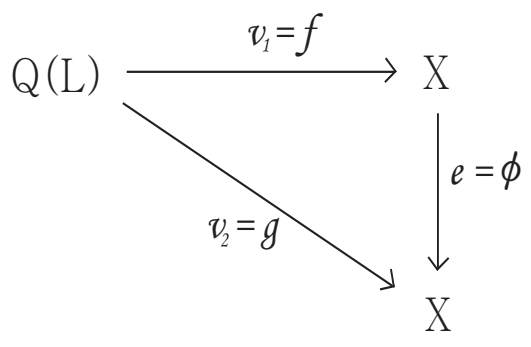

In [1, we find the following result:

Theorem 2. Let $X$ be a finite quandle, $S \subset \operatorname{Hom}(X, X)$ and $L$ an oriented link. Then the quiver $Q_{X}^{S}(L)$ is an invariant of $L$.

Corollary 3. Any invariant of directed graphs applied to $Q_{X}^{S}(L)$ defines an invariant of oriented links.

Example 7. Let $X$ be the quandle with operation table

\begin{tabular}{c|cccccc}
$\triangleright$ & 1 & 2 & 3 & 4 & 5 & 6 \\
\hline 1 & 1 & 3 & 2 & 1 & 1 & 1 \\
2 & 3 & 2 & 1 & 2 & 2 & 2 \\
3 & 2 & 1 & 3 & 3 & 3 & 3 \\
4 & 5 & 5 & 5 & 4 & 4 & 5 \\
5 & 4 & 4 & 4 & 5 & 5 & 4 \\
6 & 6 & 6 & 6 & 6 & 6 & 6.
\end{tabular}

The endomorphism ring $\operatorname{Hom}(X, X)$ has 68 elements including for example $\phi=[6,6,6,5,4,2]$, i.e., the map sending 1 to $\phi(1)=6,2$ to $\phi(2)=6, \ldots, \phi(6)=2$. Then the trefoil knot $3_{1}$ has quandle coloring quiver $Q_{X}^{\phi}\left(3_{1}\right)$ given by

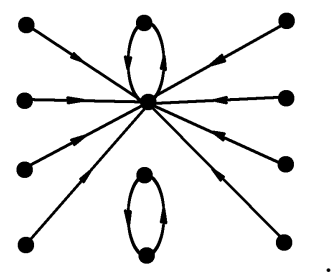

Example 8. The full quandle coloring quiver for the $(4,2)$-torus link with respect to the quandle given by the operation table

\begin{tabular}{l|lll}
$\triangleright$ & 1 & 2 & 3 \\
\hline 1 & 1 & 1 & 2 \\
2 & 2 & 2 & 1 \\
3 & 3 & 3 & 3
\end{tabular}




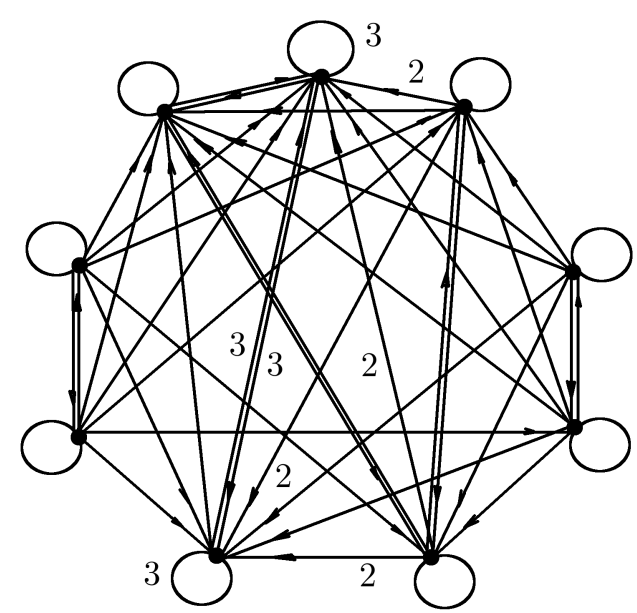

where we indicate multiple edges with numbers.

As noted in [1], the out-degree $\mathrm{deg}^{-}(f)$ of every vertex is $|S|$; however, different vertices may have different in-degrees $\mathrm{deg}^{+}(f)$. In-degrees of each vertex can be encoded as a polynomial knot invariant:

Definition 13. Let $X$ be a finite quandle, $S \subset \operatorname{Hom}(X, X)$ a set of quandle endomorphisms, $L$ an oriented link and $Q_{X}^{S}(L)$ the associated quandle coloring quiver with set of vertices $V\left(Q_{X}^{S}(L)\right)$. Then the in-degree quiver polynomial of $L$ with respect to $X$ is

$$
\Phi_{X, S}^{\operatorname{deg}^{+}}(L)=\sum_{f \in V\left(Q_{X}^{S}(L)\right)} u^{\operatorname{deg}^{+}(f)} .
$$

If $S=\{\phi\}$ is a singleton we will write $V\left(Q_{X}^{S}(L)\right)$ as $V\left(Q_{X}^{\phi}(L)\right)$ and $\Phi_{X, S}^{\mathrm{deg}^{+}}(L)$ as $\Phi_{X, \phi}^{\mathrm{deg}^{+}}(L)$, and and if $S=H o m(X, X)$ we will write $V\left(Q_{X}^{S}(L)\right)$ as $V\left(Q_{X}(L)\right)$ and $\Phi_{X, S}^{\mathrm{deg}^{+}}(L) \operatorname{as}_{X}^{\mathrm{deg}^{+}}(L)$.

Example 9. In Example 8, the $(4,2)$-torus link $L 4 a 1$ has in-degree quiver polynomial $\Phi_{X, \phi}^{\mathrm{deg}^{+}}(L)=5+u+$ $2 u^{2}+u^{4}$ with respect to the quandle $X$ and endomorphism $\phi=[1,1,2]$.

We will now extend quandle coloring quivers to the case of surface-links.

Definition 14. Let $\mathrm{X}$ be a quandle and let $D$ be an oriented marked graph diagram. Let $A(D)$ be the set of arcs of $D$. A coloring of $D$ by $X$, also called an $X$-coloring of $D$, is an assignment of elements of $X$ to the elements of $A(D)$ as shown:
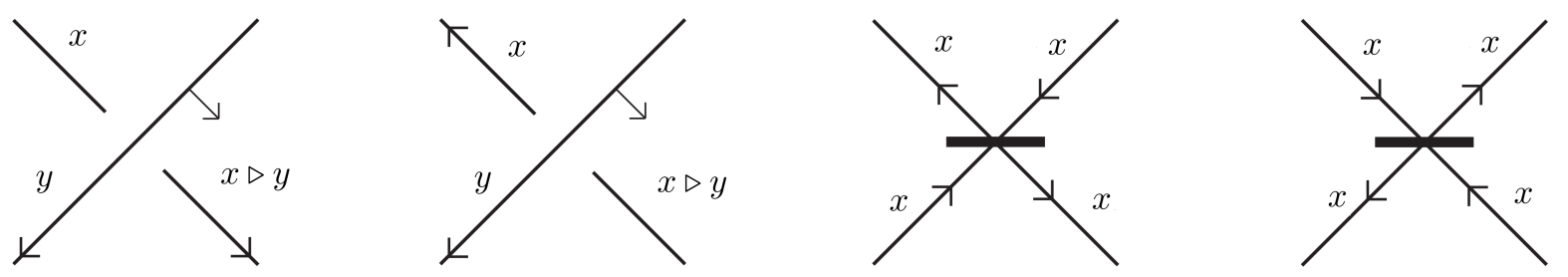

We denote by $\operatorname{Col}_{X}(D)$ the set of all X-colorings of D. 
Theorem 4. Let $X$ be a finite quandle, $L$ an oriented surface-link and $D$ a marked graph diagram of $L$. Then,

$$
\sharp \operatorname{Col}_{X}(L)=\sharp \operatorname{Col}_{X}(D)
$$

We now have our main theorem:

Theorem 5. Let $X$ be a finite quandle, $S \subset \operatorname{Hom}(X, X)$ and $L$ an oriented surface-link. Then the quiver $Q_{X}^{S}(L)$ is an invariant of $L$.

Proof. As noted in [3] and elsewhere, the set of quandle colorings is preserved by oriented Yoshikawa moves. Then as noted in [1], the quandle coloring quiver is determined by the set of quandle homomorphisms from the fundamental quandle of the surface-link to the coloring quandle.

Example 10. Let $X$ be the quandle given by the operation table

\begin{tabular}{l|llll}
$\triangleright$ & 1 & 2 & 3 & 4 \\
\hline 1 & 1 & 1 & 4 & 3 \\
2 & 2 & 2 & 2 & 2 \\
3 & 4 & 3 & 3 & 1 \\
4 & 3 & 4 & 1 & 4
\end{tabular}

and let $\phi=[2,4,2,2]$ be the quandle endomorphism mapping $1,3,4 \in X$ to $2 \in X$ and mapping $2 \in X$ to $4 \in X$. Then the surface-links $6_{1}^{0,1}$ and $8_{1}$ both have ten $X$-colorings, but are distinguished by their quandle colorings quivers as shown:
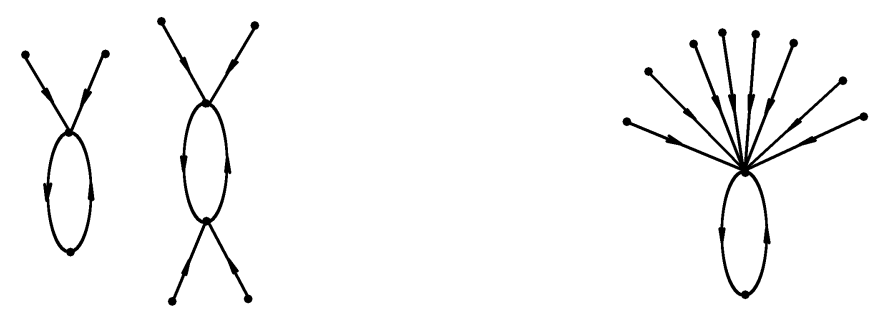

$$
Q_{X}^{\phi}\left(6_{1}^{0,1}\right)
$$

$$
Q_{X}^{\phi}\left(8_{1}\right)
$$

In particular, this example shows that the quandle coloring quiver of a surface link is not determined by the number of quandle colorings of the surface-link and hence is a proper enhancement.

Corollary 6. Let $X$ be a finite quandle, $S \subset \operatorname{Hom}(X, X)$ and $L$ an oriented surface-link. Then the in-degree polynomial $\Phi_{X}^{\operatorname{deg}_{+}}(L)$ is an invariant of $L$.

\begin{tabular}{|c|c|c|c|c|c|c|c|c|c|c|c|c|c|}
\hline$R$ & 1 & $?$ & 3 & $\triangleright$ & 1 & 2 & 3 & 4 & 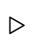 & 1 & 2 & 3 & 4 \\
\hline 1 & 1 & $\frac{2}{3}$ & 2 & 1 & 1 & 3 & 1 & 3 & 1 & 1 & 4 & 2 & 3 \\
\hline$?$ & 3 & ? & 1 & 2 & 4 & 2 & 4 & 2 & 2 & 3 & 2 & 4 & 1 \\
\hline 3 & 2 & 1 & 3 & 3 & 3 & 1 & 3 & 1 & 3 & 4 & 1 & 3 & 2 \\
\hline & & & & 4 & 2 & 4 & 2 & 4, & 4 & 2 & 3 & 1 & 1. \\
\hline
\end{tabular}

Example 11. Let $X$ be the dihedral 3-quandle, $Y$ the dihedral 4-quandle and $Z$ the tetrahedral quandle given by the operation tables as shown below.

Let $L$ be an oriented surface-link with ch-index $\chi(L) \leq 10$ presented by marked graph diagrams as shown below. 

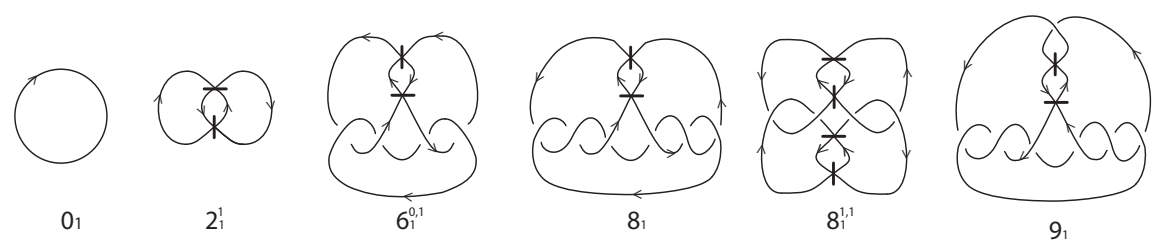

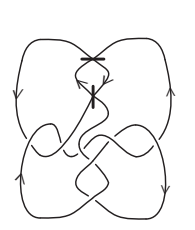

$9_{1}^{0,1}$

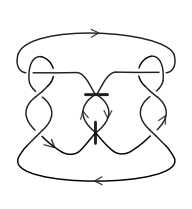

101

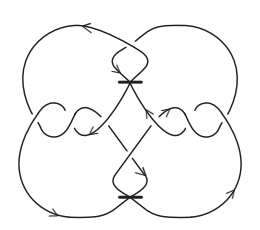

$10_{2}$

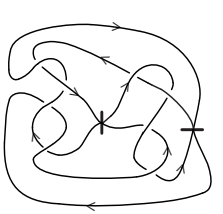

$10_{3}$

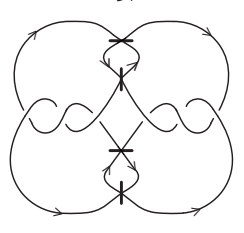

10

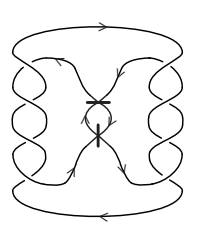

$10_{1}^{0,1}$

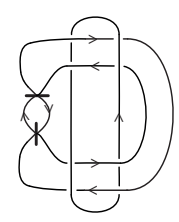

$10_{2}^{0,1}$

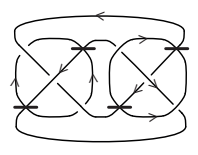

10,1

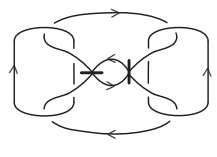

$10_{1}^{0,0,1}$

Then for each $L$, we have in-degree quiver polynomials $\Phi_{X}^{d e g^{+}}(L), \Phi_{Y}^{d e g^{+}}(L)$ and $\Phi_{Z}^{d e g^{+}}(L)$ as follows:

\begin{tabular}{|l|l|l|l|}
\hline$L$ & $\Phi_{X}^{\text {deg }^{+}}(L)$ & $\Phi_{Y}^{\text {deg }^{+}}(L)$ & $\Phi_{Z}^{\text {deg }^{+}}(L)$ \\
\hline $0_{1}$ & $3 u^{9}$ & $4 u^{16}$ & $4 u^{16}$ \\
\hline $2_{1}^{2}$ & $3 u^{9}$ & $4 u^{16}$ & $4 u^{16}$ \\
\hline $6_{1}^{0,1}$ & $3 u^{9}$ & $4 u^{8}+4 u^{24}$ & $4 u^{16}$ \\
\hline $8_{1}$ & $6 u^{6}+3 u^{12}$ & $4 u^{16}$ & $12 u^{12}+4 u^{28}$ \\
\hline $8_{1}^{1,1}$ & $3 u^{9}$ & $4 u^{8}+4 u^{24}$ & $4 u^{16}$ \\
\hline $9_{1}$ & $6 u^{6}+3 u^{12}$ & $4 u^{16}$ & $4 u^{16}$ \\
\hline $9_{1}^{0,1}$ & $3 u^{9}$ & $8 u^{8}+4 u^{16}+4 u^{32}$ & $4 u^{16}$ \\
\hline $10_{1}$ & $3 u^{9}$ & $4 u^{16}$ & $12 u^{12}+4 u^{28}$ \\
\hline $10_{2}$ & $6 u^{6}+3 u^{12}$ & $4 u^{16}$ & $4 u^{16}$ \\
\hline $10_{3}$ & $3 u^{9}$ & $4 u^{16}$ & $4 u^{16}$ \\
\hline $10_{1}^{1}$ & $6 u^{6}+3 u^{12}$ & $4 u^{16}$ & $12 u^{12}+4 u^{28}$ \\
\hline $10_{1}^{0,1}$ & $3 u^{9}$ & $8 u^{8}+4 u^{16}+4 u^{32}$ & $12 u^{12}+4 u^{28}$ \\
\hline $10_{2}^{0,1}$ & $6 u^{6}+3 u^{12}$ & $8 u^{8}+4 u^{16}+4 u^{32}$ & $4 u^{16}$ \\
\hline $10_{1}^{1,1}$ & $3 u^{9}$ & $4 u^{8}+4 u^{24}$ & $4 u^{16}$ \\
\hline $10_{1}^{0,0,1}$ & $6 u^{6}+3 u^{12}$ & $24 u^{8}+4 u^{24}+4 u^{52}$ & $12 u^{12}+4 u^{28}$ \\
\hline
\end{tabular}

We conclude this section with an observation:

Remark 2. We observe that a coloring of a marked graph diagram is also a coloring of the top and bottom smoothed diagrams $T$ and $B$. It follows that for any cobordism $L$ from $T$ to $B$, the quandle coloring quiver of $L$ is a subquiver of the quandle coloring quivers of $T$ and $B$ respectively.

\section{Questions}

In this paper, we have only initiated the study of quandle coloring quiver invariants of surface-links via marked graph diagrams. In this section we collect some open questions for future work in this area. 
- Can the observation in Remark 2 be applied to find obstructions to knot concordance or cobordisms?

- For which quandles is the quandle coloring quiver of cobordism precisely the intersection of the quivers of the top and bottom smoothed diagrams?

- What other properties of quandle-colored marked graph diagrams can be used to enhance the quandle coloring quiver?

- Since a quiver is a category, the quandle coloring quiver is a categorification of the quandle coloring invariant of oriented surface-links. What categorical properties do these categories have for particular classes of quandles, for a choice of surface-link?

\section{References}

[1] K. Cho and S. Nelson. Quandle coloring quivers. J. Knot Theory Ramifications, 28(1):1950001, 12, 2019.

[2] M. Elhamdadi and S. Nelson. Quandles - an introduction to the algebra of knots, volume 74 of Student Mathematical Library. American Mathematical Society, Providence, RI, 2015.

[3] J. Kim, Y. Joung, and S. Y. Lee. On the Alexander biquandles of oriented surface-links via marked graph diagrams. J. Knot Theory Ramifications, 23(7):1460007, 26, 2014.

[4] J. Kim, Y. Joung, and S. Y. Lee. On generating sets of Yoshikawa moves for marked graph diagrams of surface-links. J. Knot Theory Ramifications, 24(4):1550018, 21, 2015.

[5] S. J. Lomonaco, Jr. The homotopy groups of knots. i. how to compute the algebraic 2-type. Pacific J. Math., 95(2):349-390, 1981.

[6] S. Nelson and W. D. Neumann. The 2-generalized knot group determines the knot. Commun. Contemp. Math., 10(suppl. 1):843-847, 2008.

Department of Mathematics,

Pusan National University,

Busan 46241, Republic of Korea

Department of Mathematical Sciences

Claremont McKenna College

850 Columbia Ave.

Claremont, CA 91711 